\title{
An Update on the Measurement and Management of Cholesterol with Specific Reference to Secondary Prevention of Cardiovascular Disease (CVD)
}

\author{
Vally M,* Pharmacology, Lecturer; Kathrada F, Pharmacology, Lecturer; Butkow N, Senior Lecturer \\ Division of Pharmacology, Department of Pharmacy and Pharmacology, School of Therapeutic Sciences \\ Faculty of Health Sciences, University of Witwatersrand, Johannesburg \\ *Corresponding author, email: muhammed.vally@wits.ac.za
}

\begin{abstract}
Cardiovascular disease remains the largest contributor to non-communicable adverse disease outcomes. Treatment and prevention of cardiovascular disease have evolved at a dramatic pace in the last 40 years. Serum-cholesterol has emerged as the dominant risk factor for coronary artery disease and events. The link between serum-cholesterol and arterial atherosclerosis is well documented. The attainment of cholesterol goals has historically concentrated on low-density lipoprotein cholesterol (LDL-C) levels. Current evidence and guidelines have shifted to the attainment of non-HDL-C target levels which represent a more thorough inclusion of small dense atherogenic particles. Methods to reduce serum-cholesterol mainly centre around the use of the HMG CoA-reductase inhibitors also known as the statins. High intensity statins like atorvastatin ( $80 \mathrm{mg})$ and rosuvastatin (40 $\mathrm{mg})$ are now the preferred starting therapies to lower cholesterol by at least $40-50 \%$ in patients with established cardiovascular disease as secondary prevention. In the event of failure of these medications, evidence suggests that the addition of ezetimibe may enhance the total serum-lowering levels to 50-60\%. New therapies aimed at inhibiting PCSK9 revealed exciting new targets for LDL-C lowering, but the high cost of these antibodies could preclude access to this therapeutic intervention. Aggressive pursuit of lower LDL-C or nonhigh-density lipoprotein cholesterol (non-HDL-C) levels may reduce the incidence of secondary myocardial infarctions, strokes and death from cardiovascular disease.
\end{abstract}

Keywords: secondary prevention, LDL-C, Non-HDL-C, high intensity statins, PSCK9 inhibitors, ezetimibe

\section{Introduction}

Patients with established cardiovascular disease (CVD) are at a higher risk for future CVD events. ${ }^{1}$ All CVD prevention guidelines describe that the patients with the most to gain from treatment are those at greatest risk of coronary heart disease (CHD). ${ }^{1}$ Prevention in high risk patients or very high risk groups is referred to as secondary prevention. ${ }^{1,2,3}$ These individuals who are at high risk include those patients with established atherosclerotic disease such as: previous myocardial infarctions (Mls), a current history of angina, recipients of coronary revascularisation, type 2 diabetes, type 1 diabetes with microalbuminuria, genetic dyslipidaemia, chronic kidney disease (GFR $<60 \mathrm{ml} / \mathrm{min} / 1.73 \mathrm{~m}^{2}$ ), a previous stroke, a history of transient ischaemic attacks (TIA) and patients with peripheral artery disease (PAD). These patients are automatically eligible for secondary prevention measures. In addition to meeting the requirements for secondary prevention, guidelines also provide specific targets for blood pressure control or total cholesterol (TC), low-density lipoprotein cholesterol (LDL-C) or non-high density lipoprotein cholesterol (Non-HDL-C). ${ }^{1,2,3}$

\section{Measurement of Cholesterol - To Fast or Not to Fast?}

Past dyslipidaemia guidelines have generally emphasised the need for fasting lipograms before starting therapy to ensure the accuracy in results. ${ }^{2}$ There is recent evidence which has questioned the fasting requirement for lipograms. ${ }^{4,5}$ From population-based studies, TC, high density lipoprotein cholesterol (HDL-C) and non-LDL-C only varied by $2 \%$ with population based studies. ${ }^{4}$ Robust evidence supports the use of non-fasting blood draws for routine clinical practice and the role of the fasting lipograms now has a much more limited use. ${ }^{5}$ The limited role of the fasting lipograms should be utilised only in the setting of abnormally increased triglyceride (TG) levels and prior to starting treatment in patients with genetic forms of dyslipidaemia. ${ }^{4,5}$ For the purposes of monitoring LDL-C, a fasting sample may still be required. ${ }^{4}$ Both the South African Diabetes Guidelines and the current EDL, have moved away from the need for a fasting lipograms to initiate statin therapy as secondary prevention. ${ }^{6,7}$ The authors of the diabetes guidelines, state at the first visit a patient with type 2 diabetes should have their total cholesterol and triglycerides measured. If either of these is elevated then only a ten-hour fast lipogram should be performed. ${ }^{6}$ 


\section{Lowering LDL-C as a goal in Secondary Prevention of CVD}

Another set of different clinical guidelines recommend lowering LDL-C as the goal of cholesterol management in secondary prevention of CVD. ${ }^{2,8,9,10,11}$ Each of these organisations have different goals with regards to $L D L-C$ reduction ranging from obtaining an LDL-C of less than $1.8 \mathrm{mmol} / \mathrm{I}$ to an LDL-C of less than $2.6 \mathrm{mmol} / \mathrm{I} .8,8,9,10,11$ Additionally, the South African guidelines suggest that for patients who cannot achieve an LDL-C goal of less than $1.8 \mathrm{mmol} / \mathrm{l}$ for secondary prevention, they must at least achieve a greater than $50 \%$ reduction in LDL-C from baseline. It is important to remember that there is no single study that has evaluated an LDL-C goal of $<1.8 \mathrm{mmol} / \mathrm{l}$ as a target for therapy. ${ }^{12,13}$ All trials of LDL-C lowering have, however, demonstrated better outcomes in groups that received more aggressive LDL-C lowering therapy when compared to either the placebo or lower intensity statins. ${ }^{12,13}$ In many trials, participants have achieved an LDL-C below $1.8 \mathrm{mmol} / .^{12,13}$

There is a lack of reliable data regarding the optimal method of monitoring the effects of lipid-lowering therapy. ${ }^{14}$ The NCEP ATP-III guidelines suggest that LDL-C should be monitored approximately six weeks after the initiation or change of treatment whilst the South African guidelines suggest that testing should commence \pm 4 weeks after the initiation of pharmacotherapy. ${ }^{2,14}$ Should the LDL-C be less than expected, the practitioner should consider issues such as possible nontolerance or non-adherence. ${ }^{12}$ Once the goal is achieved, the follow-up testing should be every 6 months. ${ }^{2,14}$

The question does remain, however, what are the benefits of lowering of LDL-C in secondary prevention of cardiovascular disease? Table 1 illustrates the benefits of lowering LDL-C.

Table 1: Benefits of lowering LDL-C ${ }^{2}$

\section{For every $\mathrm{mmol} / \mathrm{I}$ reduction in LDL-C there is a:}

1. $10 \%$ reduction in mortality

2. $20 \%$ reduction in all-cause morbidity

3. $23 \%$ reduction in major cardiac events

4. $17 \%$ reduction in stroke.

\section{Non-High-Density Lipoprotein-Cholesterol (Non-HDL-C) as a goal in Secondary prevention in CVD}

It is generally accepted that high levels of LDL-C play the main role in the initiation and progression of atherosclerosis. ${ }^{15}$ However, despite therapies being available to reduce LDL-C a large number of clinical events still occur in patients who have reached LDL-C goals. ${ }^{16}$ This is where non-HDL-C comes into the picture. Non-HDL-C represents the cholesterol content in all the atherogenic lipoproteins and not just the LDL-C. ${ }^{16}$ Virani therefore goes on to suggest that treatment of non-HDL-C is a more grounded and holistic approach to the management of dyslipidaemia than simply targeting a particular LDL-C level. ${ }^{16}$ Non-HDL-C is calculated as follows: Non-HDL-C = Total Cholesterol - HDL-C. ${ }^{3}$
The advantage of using non-HDL-C is that it does not require a fasting sample and can be done at any time. ${ }^{3} \mathrm{~A}$ meta-analysis of 68 studies show that non-HDL-C was a better predictor when compared to all other cholesterol measurements for both coronary artery disease (CAD) events and strokes. ${ }^{17}$ Elevated levels of non-HDL-C, when found in combination with normal levels of LDL-C, can identify a subset of patients with elevated LDL particle numbers, elevated apoprotein B concentrations and LDL of small and dense morphology. ${ }^{16}$ With increased incidence of the metabolic syndrome, there might be a decrease in the accuracy of LDL-C to predict CAD events, whereas nonHDL-C, total apoprotein B concentrations and LDL particle concentrations retain their predictability in this population. ${ }^{16}$ Non-HDL-C is treatable using all the treatment modalities available for dyslipidaemia. ${ }^{16}$

Compared to other guidelines around the world, the National Institute of Health Care and Excellence (NICE) saw fit in 2014 to adopt non-HDL-C instead of LDL-C as the goal for dyslipidaemia management. $2,3,6-10,13$ NICE suggests that if a patient has established cardiovascular disease, they should be started on a high-intensity statin, e.g. atorvastatin $80 \mathrm{mg}^{3}$ After three months on this regimen, the authors suggest a lipid profile must be obtained. In this situation total cholesterol, HDL-C and nonHDL-C should be measured and the goal of therapy is to have reduced non-HDL-C by more than $40 \% .^{3}$ If this is not obtained they suggest the practitioner should institute one of the measures stated in table 2.

Table 2: Suggested measures primary care practitioners should take if a patient has not achieved a $40 \%$ reduction from baseline non-HDL-C

1. Discuss adherence and timing of dose with the patient.

2. Optimise adherence to diet and lifestyle measures.

3. Consider increasing dose if started on less than atorvastatin $80 \mathrm{mg}$ and the person is judged to be at higher risk because of comorbidities, risk score or using clinical judgement.

It remains unclear whether the goal of lipid-lowering therapy in secondary prevention of CVD should be centred on LDL-C or non-HDL-C. It is clear, however, that non-HDL-C may be an alternative goal to LDL-C.

\section{Pharmacological therapy}

For secondary prevention patients, high-intensity statin therapy is recommended, which is consistent with the NICE guidelines, ATP-III guidelines, South African guidelines and European guidelines. . $3,8,8,9,14$ High-intensity statin therapy is expected to lower LDL-C by $50-60 \%$. Combination therapy (a statin plus ezetimibe) is recommended in cases where the LDL goal is not met with statin therapy alone. ${ }^{2,3}$ If a compliant patient's LDL goal is still not met with combination therapy of a statin with ezetimibe, referral to a cardiologist specialist in lipid disorders is necessary for the possible addition of PCSK9 antibody therapy. ${ }^{3}$

\section{Statin therapy}

The principle therapeutic benefits of statins derive from their ability to reduce cholesterol low-density lipoprotein (LDL) 
by inhibiting 3-hydroxy-3-methyl-glutaryl-CoA (HMG COA) reductase enzymes. This results in lower intrahepatic cholesterol and an up-regulation of cell surface LDL receptors, resulting in enhanced receptor-mediated uptake of LDL and other apoBcontaining lipoproteins from circulation. ${ }^{18}$ The benefit of statin therapy in reducing cardiovascular events in patients with known atherosclerotic cardiovascular disease has been wellestablished. 19,20,21 In addition, more intensive statin regimens have been found to have a greater efficacy compared to lessintensive regimens. According to the NICE guidelines, secondary prevention is started with a high intensity statin (atorvastatin $80 \mathrm{mg}$ ) unless the patient experiences high incidence of adverse effects, are on interaction drugs or patient preference. ${ }^{3}$ If a patient presents with statin intolerance, it is best to treat with the maximum tolerable dose or change over to a different statin. A meta-analysis involving approximately 170000 patients on statin therapy, found that there was an $18 \%$ risk reduction for fatal myocardial infarction and $26 \%$ reduction in non-fatal myocardial infarction. ${ }^{22}$ The CTT meta-analysis demonstrated that $1 \mathrm{mmol} / \mathrm{l}$ reduction in LDL-C resulted in a $10 \%$ relative reduction in all-cause mortality and $21 \%$ reduction in MVE for statins versus placebo. ${ }^{23}$ Table 3 classifies the potency of different statins.

Table 3: The classification of statin potency ${ }^{3}$

\begin{tabular}{lll}
\hline High intensity & Medium Intensity & Low Intensity \\
\hline Atorvastatin 20-80 mg & Fluvastatin $80 \mathrm{mg}$ & Fluvastatin 20-40 mg \\
Simvastatin $80 \mathrm{mg}$ & Simvastatin 20-40 mg & Pravastatin 10-40 mg \\
Rosuvastatin 10-40 mg & Atorvastatin $10 \mathrm{mg}$ & Simvastatin 10 mg \\
& Rosuvastatin $5 \mathrm{mg}$ & \\
\hline
\end{tabular}

While the benefits of statins are undisputed, they have also been evaluated for potential long-term adverse effects. Adverse event data reported excess risk of myopathy of 0.5 per 1000 in statin therapy trials and was associated with $80 \mathrm{mg}$ rather than $20 \mathrm{mg}$ simvastatin use. ${ }^{24}$ It has been proposed that higher potency statins at standard doses could help patients attain their treatment goals without increasing the risk of myopathy. ${ }^{25}$ Statins are associated with an increased risk of new-onset diabetes, with adverse event data in more moderate intensity statin trials reported 5 per 1000 diabetogenic potential. ${ }^{26}$ Statins have also been associated with increasing the risk of hepatic injury which occurs rarely in $1 \%$ of patients and is reversible with dose reduction or discontinuation. ${ }^{27,28}$ Higher doses of statins confer significant CVD benefits, but a higher risk of adverse events. Table 4 illustrates the trials related the combination of statins and PCSK9 inhibitors, statins and ezetimibe as well as comparator statin intensity trials all in the secondary prevention of CVD.

\section{Second-line therapy: Ezetimibe}

Ezetimibe inhibits the function of the NPC1L1 protein which is responsible for the transportation of dietary cholesterol from the gut lumen to intestinal enterocytes, thus reducing the absorption of intestinal enterocytes. ${ }^{36,37}$ Combination therapy of ezetimibe with statin therapy compared to statin therapy by itself modestly decreases the risk of cardiovascular disease events, but not mortality in high-risk patients with an acute coronary syndrome. Findings from the IMPROVE-IT trial suggest that the ezetimibe-induced cardiovascular risk reduction by $\mathrm{LDL}-\mathrm{C}$ reduction is similar to statins. ${ }^{38}$ Ezetimibe is the first LDL-C lowering drug to show a reduction in $\mathrm{CV}$ outcomes in patients well-treated with statins. ${ }^{18}$

\section{Third-line therapy: PCSK9 inhibitors}

In certain patients, satisfactory control of dyslipidaemia is not achieved even with combination lipid lowering therapy and recent attention has focused on a new class of agents, proprotein convertase subtilisin/kexin type 9 (PCSK9) inhibitors. ${ }^{3}$ These agents provide great promise for patients who require additional LDL lowering or are unable to take statins (statin-intolerant and familial hypercholesterolemia). ${ }^{3}$ The PCSK9 protein plays a crucial role in the regulation of $L D L$ receptors by reducing the number of LDL-C receptors. ${ }^{39}$ Targeted monoclonal antibodies have the ability to bind to PCSK9, thereby inhibiting its interaction with LDL cholesterol receptors. ${ }^{39}$ Outcomes with regard to their efficacy indicate a reduction in LDL cholesterol of greater than $50 \%$ and an elevation in HDL cholesterol levels, especially when administered with statin therapy. ${ }^{40}$ In clinical trials the two PCSK9 inhibitors that have shown significant promise are evolocumab and alirocumab. ${ }^{41}$

It is clear that high intensity statins provide benefit irrespective of the baseline LDL-C levels in the secondary prevention of cardiovascular events. ${ }^{2,9-11,14}$ Statins by their nature increase PSCK9 levels(Table 5). ${ }^{42-45}$ It is evident that PCSK9 inhibitors modulate the effects of statins leading to an increased reduction in LDL-C levels demonstrated by clinical trials such as ODYSSEY LONG TERM. ${ }^{30}$

Table 5: The impact of statin therapy on PCSK9 $9^{42-45}$

\begin{tabular}{lc}
\hline Statin & PCSK change \\
\hline Atorvastatin $80 \mathrm{mg}$ & $+47 \%$ \\
\hline Atorvastatin $40 \mathrm{mg}$ & $+37 \%$ \\
Rosuvastatin $20 \mathrm{mg}$ & $+28 \%$ (men) \\
& $+35 \%$ (women) \\
Controls & \\
Statin therapy & $+45 \%$ \\
Statin Ezetimibe therapy & $+77 \%$ \\
Titration of atorvastatin 5 to $80 \mathrm{mg} /$ day & $+30 \%$ \\
Titration of atorvastatin 5 to $40 \mathrm{mg} /$ day & $+37 \%$ \\
\hline
\end{tabular}

The recent ODYSSEY LONG TERM study (a post-hoc analysis of the ODYSSEY trial) demonstrated that alirocumab resulted in a $62 \%$ LDL-C reduction from baseline and the incidence of major cardiovascular events reduced by $48 \% .^{30}$ The OSLER studies evaluated demonstrated that evolocumab plus high intensity statin therapy reduced LDL-C by $61 \%$ and the incidence of major cardiovascular events by 53\%.32 PCSK9 inhibition was well-tolerated without excess of new-onset diabetes or neurocognitive effects with the exception of a $2 \%$ incidence of injection site reactions, despite dramatic LDL reduction. In the OSLER and ODYSSEY LONG TERM studies, the rate of any adverse events was similar in patients receiving PCSK9 inhibitors compared to placebo. ${ }^{30,32}$ The recently announced Odyssey Outcome Trial, demonstrated that in 18000 patients with recent 


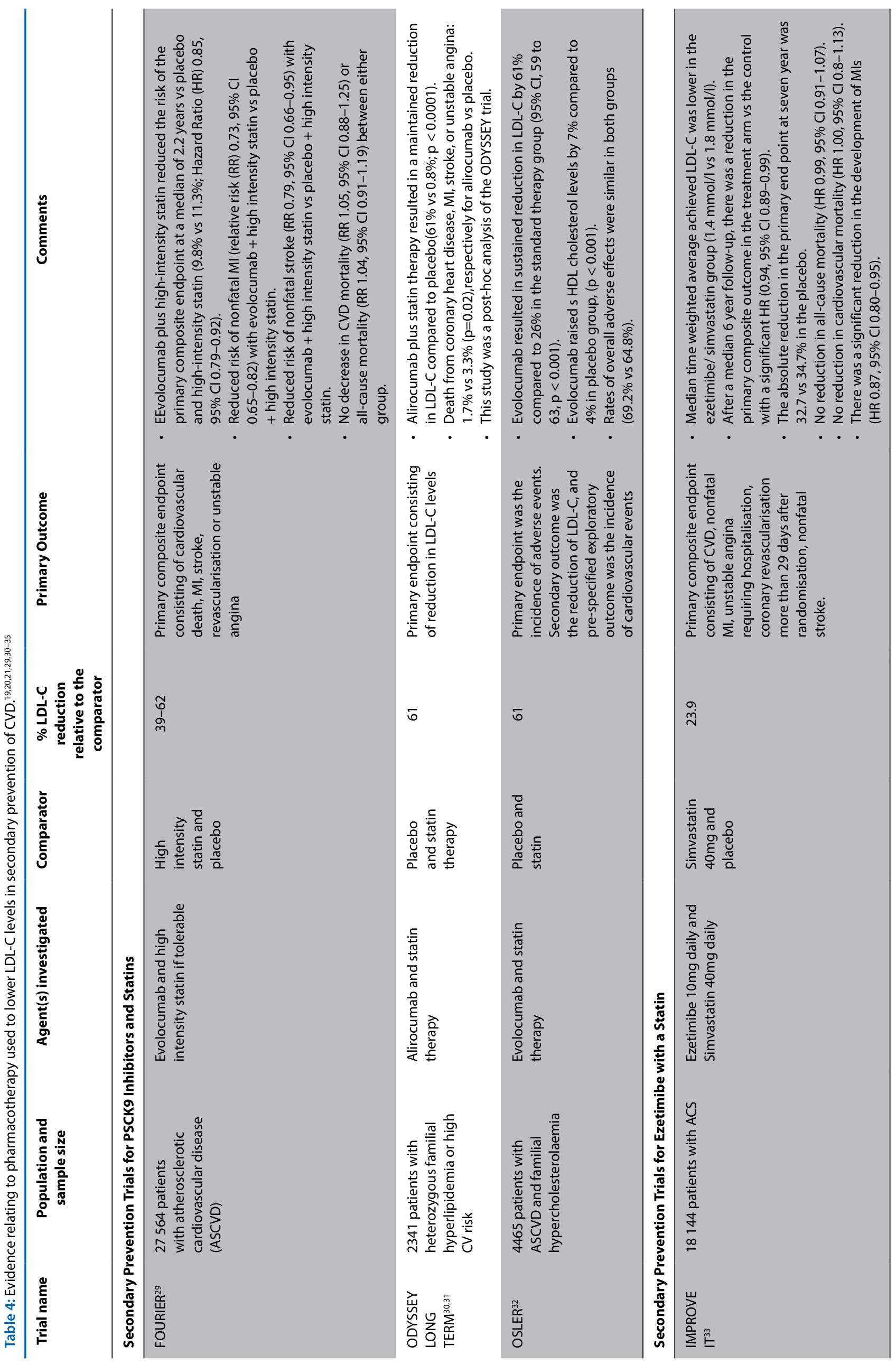




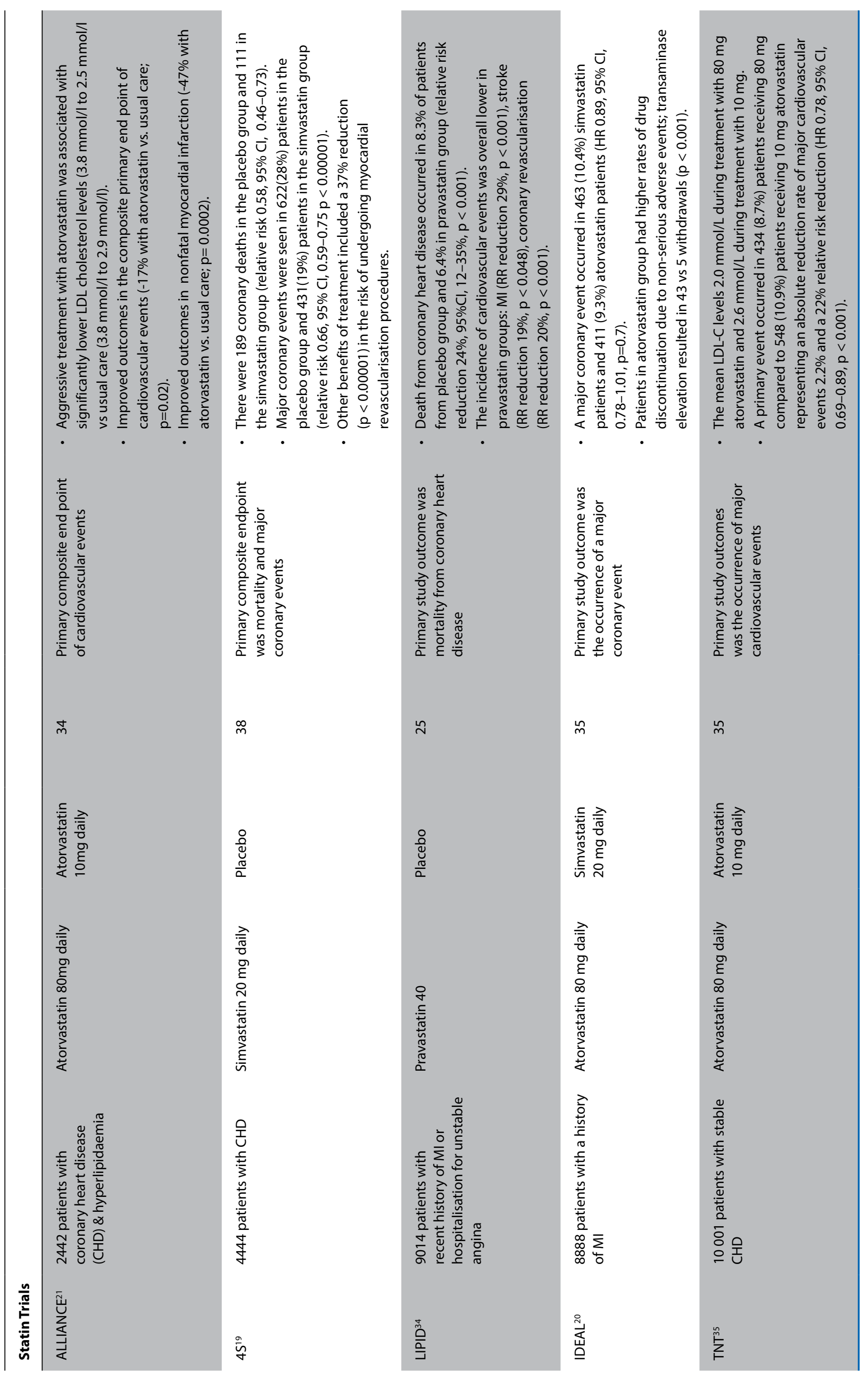


ACS, alirocumab and high intensity statin therapy significantly reduced measures of acute and/or adverse cardiovascular outcomes (MACE) compared to the placebo. ${ }^{46}$ The goal of the trial was to reduce patient's serum LDL-C to between $0.65 \mathrm{mmol} / \mathrm{I}$ and $1.30 \mathrm{mmol} / \mathrm{l}$ which was much lower than the current goals in patients with established coronary artery disease. The trial met its primary endpoint of a $15 \%$ hazard reduction (HR $0.85,95 \% \mathrm{Cl}$ 0.78-0.93, $p=0.0003$ ) in the composite outcome of CHD death, nonfatal $\mathrm{MI}$, fatal or nonfatal ischaemic stroke and unstable angina and thus the trial was considered a success. Therapy with alirocumab and high intensity statins resulted in significant hazard reductions in nonfatal $\mathrm{MI}(\mathrm{HR} 0.86,95 \% \mathrm{Cl} 0.77-0.96$, $\mathrm{p}=0.006$ ), ischaemic stroke ( $\mathrm{HR} 0.73,95 \% \mathrm{Cl} 0.57-0.93, \mathrm{p}=0.01$ ) and unstable angina $(\mathrm{HR} 0.61,95 \% \mathrm{Cl} 0.41-0.92, \mathrm{p}=0.02)$ at 4 years. However, patients in the treatment group experienced no significant hazard reduction in CHD death when compared to the placebo. In the case of both MACE and all-cause mortality a difference in the Kaplan-Meier was only seen after one year. Subgroup analysis of this study indicates that patients with serum LDL-C level consistently greater than $2.59 \mathrm{mmol} / \mathrm{l}$ benefited most from this therapy 4 years after randomiation. ${ }^{46}$

PCSK9 inhibitors show promising features alone or in combination in patients with unmet LDL-C goals, statin intolerance or heterozygous familial hyperlipidaemia. ${ }^{3}$ However, being a monoclonal antibody, it has higher cost implications in comparison to current therapies and the fact that it is a subcutaneously administered agent may lead to patient dosing inconsistencies.

\section{Conclusion}

Treatment of cholesterol in cardiovascular disease is mainly confined to the use of the statins. Research has indicated that a preferred target for therapy maybe non-HDL-C which might be a better predictor of cardiovascular events compared to LDL-C. The statins ranging from low intensity fluvastatin to high intensity rosuvastatin have demonstrated the relationship between cholesterol reductions linked to a decrease or a reduction in fatal and non-fatal cardiovascular events. Guidelines have suggested that if cholesterol targets are not reached, the addition of a GITcholesterol blocker like ezetimibe should be considered. Since statins increase PCSK9 levels, their effect is modulated in this manner. Thus, the addition of PSCK9-inhibitors to statins can dramatically reduce serum LDL levels to as low as $0.4 \mathrm{mmol} / \mathrm{l}$, however the cost of this new technology means that a majority of patients will not have access to this class of drugs. As a result, statins remain the first and most affordable class of drugs to reduce cardiovascular mortality and morbidity.

\section{References:}

1. Hobbs FD. Cardiovascular disease: different strategies for primary and secondary prevention? Heart. Oct 2004;90(10):1217-23. Review. PubMed PMID: 15367530; PubMed Central PMCID: PMC1768505.

2. Klug E; South African Heart Association (SA Heart); Lipid and Atherosclerosis Society of Southern Africa (LASSA). South African dyslipidaemia guideline consensus statement. S Afr Med J. 23 Feb 2012;102(3 Pt 2):178-87. PubMed PMID: 22380916

3. National Institute of Care and Excellence, United Kingdom. Lipid modification Cardiovascular risk assessment and the modification of blood lipids for the primary and secondary prevention of cardiovascular disease: Clinical guideline Methods, evidence and recommendations. 2014 [Accessed on 1 March 2018]. Adapted from: NICE clinical guideline CG181. Available from: https://www.nice.org.uk/guidance/cg181/evidence/ lipid-modification-update-full-guideline-243786637

4. Naugler C, Sidhu D. Break the fast? Update on patient preparation for cholesterol testing. Can Fam Physician. Oct 2014;60(10):895-7, e471-4. Review. English, French. PubMed PMID: 25316740; PubMed Central PMCID: PMC4196810.

5. Farukhi Z, Mora S. Re-assessing the role of non-fasting lipids; a change in perspective. Ann TransI Med. Nov 2016;4(21):431. PubMed PMID: 27942522; PubMed Central PMCID: PMC5124629.

6. The Society for Endocrinology, Metabolism and Diabetes of South Africa Type 2 Diabetes Guidelines Expert Committee. The 2017 SEMDSA Guideline for the Management of Type 2 Diabetes Guideline Committee. JEMDSA. 2017;21(1) (Suppl 1):S1-S196.

7. National Department of Health, South Africa. STEMI/ N-STEMI Guidelines. 2015 [Accessed on 1 March 2018]. Adapted from: Standard Treatment Guidelines and Essential Drugs List PHC (2014) Available from: http://www.kznhealth.gov.za/ pharmacy/edlphc2014a.pdf

8. Catapano AL, Graham I, De Backer G, et al. 2016 ESC/EAS Guidelines for the Management of Dyslipidaemias. Eur Heart J. 2016;37:2999.

9. Expert Dyslipidemia Panel, Grundy SM. An International Atherosclerosis Society Position Paper: global recommendations for the management of dyslipidemia. J Clin Lipidol. 2013;7:561.

10. Anderson TJ, Grégoire J, Hegele RA, et al. 2012 update of the Canadian Cardiovascular Society guidelines for the diagnosis and treatment of dyslipidemia for the prevention of cardiovascular disease in the adult. Can J Cardiol. 2013;29:151.

11. Jellinger PS, Handelsman $Y$, Rosenblit PD, et al. American association of clinical endocrinologists and American college of endocrinology guidelines for management of dyslipidemia and prevention of cardiovascular disease - executive summary. Complete Appendix to Guidelines. Endocr Pract 2017;23:479. Available from: http://journals.aace.com

12. Boekholdt SM, Hovingh GK, Mora $S$, et al. Very low levels of atherogenic lipoproteins and the risk for cardiovascular events: a meta-analysis of statin trials. J Am Coll Cardiol. 2014;64:485.

13. Silverman MG, Ference BA, Im K, et al. Association Between Lowering LDL-C and Cardiovascular Risk Reduction Among Different Therapeutic Interventions: A Systematic Review and Meta-analysis. JAMA. 2016;316:1289.

14. National Cholesterol Education Program (NCEP) Expert Panel on Detection, Evaluation, and Treatment of High Blood Cholesterol in Adults (Adult Treatment Panel III). Third Report of the National Cholesterol Education Program (NCEP) Expert Panel on Detection, Evaluation, and Treatment of High Blood Cholesterol in Adults (Adult Treatment Panel III) final report. Circulation. 2002;106:3143.

15. Badimon L, Vilahur G. LDL-cholesterol versus HDL-cholesterol in the atherosclerotic plaque: inflammatory resolution versus thrombotic chaos. Ann $\mathrm{N}$ Y Acad Sci. Apr 2012;1254:18-32. doi: 10.1111/j.1749-6632.2012.06480.x Review. PubMed PMID: 22548566.

16. Virani SS. Non-HDL cholesterol as a metric of good quality of care: opportunities and challenges. Tex Heart Inst J. 2011;38(2):160-2. PubMed PMID: 21494527; PubMed Central PMCID: PMC3066801.

17. Emerging Risk Factors Collaboration, Di Angelantonio E, Sarwar N, Perry $P_{1}$ et al. Major lipids, apolipoproteins, and risk of vascular disease. JAMA. 11 Nov 2009;302(18):1993-2000. PubMed PMID: 19903920; PubMed Central PMCID: PMC3284229. doi: 10.1001/jama.2009.1619

18. Wadhera RK, Mphil MD, Steen DL, et al. A review of low-density lipoprotein cholesterol, treatment strategies, and its impact on cardiovascular disease morbidity and mortality. J Clin. Lipidol. June 2016; 10:172-489.

19. Pederson TR, Olsson AG, Faergeman O, et al. Lipoprotein changes and reduction in the incidence of major coronary heart disease events in the Scandanavian Simvastatin Survival Study (4S). Circulation. 1998;97:1453-60.

20. Pederson TR, Faergeman 0 , Kastelein JJ, et al. High-dose atorvastatin vs usual-dose simvastatin for secondary prevention after myocardial infarction: the IDEAL study: a randomized control trial. JAMA. 2005;94:2437-45.

21. Koren MJ, Hunninghake DB, ALLIANCE Investigators. Clinical outcomes in managed-care patients with coronary heart disease treated aggressively in lipid-lowering disease management clinics: the alliance study. J Am Coll Cardiol. 2004;44:1772-9. 
22. Mills $E$, Wu $P$, Chong $G$, et al. Efficacy and safety of statin treatment for cardiovascular disease: a network meta-analysis of 170255 patients from 76 randomized trials. QJM. 2011;104(2):109-124.

23. Cholesterol Treatment Trialists Collaboration, Baigent C, Blackwell L, Emberson $J$, et al. Efficacy and safety of more intensive lowering of LDL cholesterol: a meta-analysis of data from 170000 participants in 26 randomised trials. Lancet. 2010;376:1670-81.

24. Cholesterol Treatment Trialists Collaboration, Emberson, JR, Kearney PM, Blackwell $L$, et al. Lack of effect of lowering LDL cholesterol on cancer: meta-analysis of individual data from 175000 people in 27 randomised trials of statin therapy. PLoS One. 2012;7:e29849.

25. Cholesterol Treatment Trialists Collaboration, Mihaylova B, Emberson JR, Blackwell $L$, et al. The effects of lowering LDL cholesterol with statin therapy in people at low risk of vascular disease: meta-analysis of individual data from 27 randomised trials. Lancet. 2012;380:581-90.

26. Sattar N, Preiss D, Murray HM, et al. Statins and risk of incident diabetes: a collaborative meta-analysis of randomised statin trials. Lancet. 2010;375:735-42.

27. Gillet RC Jnr, Norrell A. Considerations for safe use of statins: liver enzyme abnormalities and muscle toxicity. Am Fam Phys. 2011;83(6):711-6.

28. Kashani A, Phillips CO, Foody JM, et al. Risks associated with statin therapy: a systematic overview of randomized clinical trials. Circulation. 2006;114:2788-97.

29. Desai NR, Sabatine MS. PCSK9 inhibition in patients with hypercholesterolemia. Trends Cardiovasc Med. 2015;25:567-74.

30. Robinson JG, Farnier M, Krempf $M$, et al. Efficacy and safety of alirocumab in reducing lipids and cardiovascular events. N Engl J Med. 2015;372:1489-99.

31. Scwartz GG, Bessac $L$, Berdan LG, et al. Effect of alirocumab, a monoclonal antibody to PCSK9, on long term cardiovascular outcomes following acute coronary syndromes: Rationale and design of ODYSSEY Outcomes trial. Am Heart J. 2014;168:682-9.

32. Sabatine MS, Giugliano RP, Wiciott SD, et al. Efficacy and safety of evolocumab in reducing lipids and cardiovascular events. N Engl J Med. 2015;372:1500-9.

33. Giugliano RP, Wiviott SD, Blazing MA, et al. Long term safety and efficacy of achieving very low levels of low-density lipoprotein cholesterol: A perspective analysis of the IMPROVE-IT trial. JAMA Cardiol. 2017;2(5):547-55.

34. The Long-Term Intervention with Pravastatin in Ischemic Disease (LIPID) Study Group. Prevention of cardiovascular events and death with pravastatin in patients with coronary heart disease and a broad range of initial cholesterol levels. N Engl J Med. 1998;339:1349-57.

35. LaRosa JC, Grundy SM, Waters DD, et al. Intensive Lipid Lowering with atorvastatin in patients with stable coronary disease. $\mathrm{N}$ Engl J Med. 2005;352:1425-35.

36. Altamann SW, Davis HR Jnr, Zhu LJ, et al. Niemann-Pick C1 Like 1 protein is critical for intestinal cholesterol absorption. Science. 2004;303:1201-4.

37. Sudhop T, Lutjohann D, Kodal A, et al. Inhibition of intestinal cholesterol absorption by ezetimibe in humans. Circulation. 2002;106:1943-8.

38. Cannon CP, Blazing MA, Giugliano RP, et al. Ezetimibe added to statin therapy after acute coronary syndromes. N Engl J Med. 2015;372:2387-97.

39. Hassan M. OSLER and ODYSSEY LONG TERM: PCSK9 inhibitors on the right track of reducing cardiovascular events. Glob Cardiol Sci Pract. 2015;2015(2):20.

40. Zhang $X$, Zhu L, Chen J, et al. Safety and efficacy of anti-PCSK9 antibodies: a meta-analysis of 25 randomised, controlled trials. BMC Med. 2015;13:123.

41. Latimer J, Batty JA, Neely RDG, Kunadian V. PCSK9 inhibitors in the prevention of cardiovascular disease. J Thromb Thrombolysis. 2016;42:405-19.

42. Welder G, Zineh I, Pacanowski MA, Troutt JS, Cao G, Konrad RJ. High-dose atorvastatin causes a rapid sustained increase in human serum PCSK9 and disrupts its correlation with LDL cholesterol. J Lipid Res. Sep 2010;51(9):271421. PubMed PMID: 20525997; PubMed Central PMCID: PMC2918453. Epub 5 Jun 2010. doi: 10.1194/jlr.M008144

43. Careskey HE, Davis RA, Alborn WE, Troutt JS, Cao G, Konrad RJ. Atorvastatin increases human serum levels of proprotein convertase subtilisin/kexin type 9. J Lipid Res. Feb 2008;49(2):394-8. Epub 21 Nov 2007. PubMed PMID: 18033751.

44. Dubuc G, Tremblay M, Paré G, Jacques $H$, Hamelin J, Benjannet S, Boulet $L$, Genest J, Bernier L, Seidah NG, Davignon J. A new method for measurement of total plasma PCSK9: clinical applications. J Lipid Res. Jan 2010;51(1):140-9. PubMed PMID: 19571328; PubMed Central PMCID: PMC2789774. doi:10.1194/ jlr.M900273-JLR200

45. Awan Z, Seidah NG, MacFadyen JG, et al. Rosuvastatin, proprotein convertase subtilisin/kexin type 9 concentrations, and LDL cholesterol response: the JUPITER trial. 2012;58(1):183-9.

46. Odyssey Outcomes Investigators and Committee [cited 10 March 2018]. The Odyssey Outcome Trial Topline results: Alirocumab in patients after Acute Coronary Syndrome [Unpublished].2018. Available at: https:// accscientificsession.acc.org/features/2018/03/video-sanofi-regeneron 